\section{Predicting macular hole closure with ocriplasmin based on spectral domain optical coherence tomography}

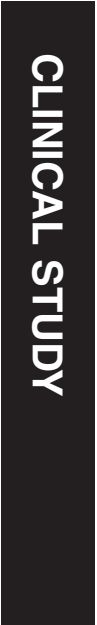

${ }^{1}$ Sunderland Eye Infirmary, Sunderland, UK

${ }^{2}$ Institute of Genetic Medicine, Newcastle University, Newcastle Upon Tyne, UK

${ }^{3}$ Newcastle Eye Centre, Royal Victoria Infirmary, Newcastle Upon Tyne, UK

${ }^{4}$ School of Mathematics and Statistics, Newcastle University, Newcastle Upon Tyne, UK

${ }^{5}$ Ophthalmology Department, James Cook University Hospital, Middlesborough, UK

Correspondence: DHW Steel, Sunderland Eye Infirmary, Queen Alexandra Road, Sunderland SR2 9HP, UK Tel: +44 (0)191 5699065; Fax: +44 (0)1915699060. E-mail: David.steel@ncl.ac.uk

Received: 3 August 2015 Accepted in revised form: 3 February 2016 Published online: 11 March 2016 MLD had an improved probability of closure than holes with wider base configurations. published online 11 March 2016
DHW Steel ${ }^{1,2}$, C Parkes $^{3}$, VT Papastavrou ${ }^{3}$, PJ Avery ${ }^{4}$, IA El-Ghrably ${ }^{5}$, MS Habib ${ }^{1}$, MT Sandinha ${ }^{1}$, J Smith ${ }^{1}$, KP Stannard ${ }^{3}$, D Vaideanu-Collins ${ }^{5}$ and RJ Hillier ${ }^{3}$

\begin{abstract}
Purpose To assess the preoperative features of patients with idiopathic macular hole (IMH) and vitreomacular adhesion (VMA) treated with ocriplasmin (OCP) that can predict successful closure.

Method Data were prospectively collected on all patients with IMH treated with OCP in three British ophthalmic centres. Several preoperative variables were recorded including the IMH base diameter (BD), minimum linear diameter (MLD), and VMA width measured on spectral domain optical coherence tomography. Several other IMH indices were derived including a 'width factor', defined as the BD minus the MLD in $\mu \mathrm{m}$. The occurrence of VMA release and hole closure were used as the main outcome measures.

Results Thirty-three patients in total with IMH were treated with OCP. Two patients developed rhegmatogenous retinal detachment and were excluded. The mean age of the remaining 31 patients was 71 years, and $71 \%$ were female. VMA release occurred in 19 of the $31(61 \%)$ patients and macular hole closure in $11(35 \%)$. Width factor was the most predictive feature for closure on multivariate analysis. The deviance $R^{2}$ was $67 \%(P<0.001)$. An IMH with a width factor of $<60 \mu \mathrm{m}$ had a $95 \%$ certainty of closure, whereas if $>290 \mu \mathrm{m}$ then there was less than a $5 \%$ chance of closure. Neither VMA width nor MLD alone was associated with VMA release or closure.

Conclusions Patients with macular holes where the BD was close in size to the

\section{Introduction}

Ocriplasmin (OCP) has been licensed for the treatment of patients with symptomatic vitreomacular adhesion (VMA) including those with full-thickness idiopathic macular holes (IMHs). The efficacy of OCP for patients with IMH and VMA was demonstrated in two phase 3 clinical trials, the Microplasmin for Intravitreous Injection-Traction Release without Surgical Treatment (MIVI-TRUST) trials, which recorded an overall FTMH closure rate of $40.6 \%$ (43 of 106 patients) in the OCP arms compared witth $10.6 \%$ (5 of 47 ) in the control vehicle injected arms. ${ }^{1}$ Closure was higher in the 48 patients with holes with a minimum linear diameter (MLD) of $<250 \mu \mathrm{m}$ at $58.3 \%$ compared with the 38 patients with medium-sized holes (250-400 $\mu \mathrm{m}$ ) at $36.8 \%{ }^{2}$ None of the 19 patients included in the study with large holes ( $>400 \mu \mathrm{m}$ ) achieved closure. ${ }^{2}$ Post-marketing studies have generally not matched these results, with lower levels of closure and less

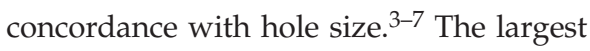
independent study to date by Sharma et al included 15 FTMH and had a $27 \%$ closure rate with $33 \%$ in small holes and $25 \%$ in mediumsized holes. ${ }^{3}$ Other features have been identified as being prognostic for VMT release with $\mathrm{OCP}$ including younger age, phakic status and absence of ERM but not specifically for macular hole closure. ${ }^{2,8}$ We prospectively collected data on patients with IMH and VMA treated with OCP to assess if any specific baseline parameters could predict successful closure.
\end{abstract}

Eye (2016) 30, 740-745; doi:10.1038/eye.2016.42;

\section{Materials and methods}

Data on all patients treated with OCP (Jetrea, Thrombogenics, Leuven, Belgium) for IMH with 
VMA during the period August 2013 to March 2015 in three large ophthalmic treatment centres clustered in the North East of England were prospectively collected using a jointly agreed data collection form. Patients were eligible for treatment with OCP as per UK National Institute for Health and Care Excellence criteria based on the presence of VMA, the width of the hole $<400 \mu \mathrm{m}$ and the absence of epiretinal membrane. (https://www.nice. org.uk/guidance/ta297; accessed 11/7/15). ${ }^{9}$ Eight surgeons carried out the treatment and all patients had $125 \mathrm{mg}$ of OCP delivered by intravitreal injection as per the manufacturer's instructions. Patients had spectral domain optical coherence tomography (SD-OCT) performed immediately pre-injection to confirm the persistence of $\mathrm{VMA}^{10}$ and from which a variety of OCT parameters were measured. The macular hole MLD in the SD-OCT slice with the maximum hole dimensions was measured using a standardised technique as described by Decroos et $a l^{11}$ as well as the base diameter (BD), height $(H)$, and maximum horizontal width $(W)$ of the VMA by one observer masked to the outcome. Two different SD-OCT devices were used: a Heidelberg Spectralis HRA+SD-OCT (Heidelberg engineering, Heidleberg, Germany) was used in two centres and a Topcon 3D SD-OCT 2000 (Topcon Corporation, Tokyo, Japan) in the other, both with high-density scanning protocols. Best corrected visual acuity, age, sex, and phakic status at baseline were also collected. Patients were assessed post injection to assess VMA release and IMH closure, defined as the absence of a full-thickness retinal defect. To be eligible for inclusion in the final analysis, patients had to have had SD-OCT performed at least 4-6 weeks post injection. Snellen visual acuities were converted to $\log$ MAR for analysis. The baseline measurements were used to calculate the following, previously described, indices; macular hole index (MHI, which is H/BD), diameter hole index (DHI, which is MLD/BD), and tractional hole index (THI, which is $H / M L D)^{12,13}$ as well as a simple calculation of the BD minus the MLD, which we termed 'width factor'. Under UK guidelines, the analysis was classified as a service evaluation and as such did not require ethical approval.

\section{Statistical analysis}

Descriptive and statistical analysis was performed using Minitab 17 (Minitab Ltd, Coventry, UK). Patients' demographic baseline characteristics are presented in terms of mean, SD, and range or percentage as appropriate. When comparing groups, mean and SE are presented. $T$-tests were used to compare continuous variables and $\chi^{2}$-tests on the discrete variables. LogMAR was dichotomised to greater or less than $0.8(6 / 36)$, as it had a limited discrete scale and the frequencies were too small to use all the categories. The derived ratios $\mathrm{MHI}$, DHI, and THI had asymmetric distributions and hence their square roots were used, which normalised them. Binary logistic regression with a stepwise option was used to distinguish the most important variables and present a predictive model.

\section{Results}

There were 33 patients (33 eyes) in total treated during the study period. Two patients experienced rhegmatogenous retinal detachment on day 5 and day 21, respectively, and were excluded from further analysis.

The baseline characteristics of the 31 included patients are shown in Table 1. All patients were white British in ethnicity. Twelve of the patients had holes with a MLD of $<250 \mu \mathrm{m}$ and 19 were between $250-400 \mu \mathrm{m}$. Overall, $19(61 \%)$ patients experienced VMA release by 1 month postoperatively and 11 (35\%) patients had hole closure. Closure occurred in 6 of the $12(50 \%)$ patients with holes with a MLD of $<250 \mu \mathrm{m}$ and 5 of the 19 (26\%) between 250 and $400 \mu \mathrm{m}$. In eight patients with VMA release, but without hole closure, five were $<250 \mu \mathrm{m}$ and three were $>250 \mu \mathrm{m}$ in MLD. There were no cases of VMA release or closure after 1 month and there were no patients who had closure without VMA release.

Several of the baseline characteristics were highly correlated (Table 2). The correlation between the baseline characteristics derived from the same variables were particularly high. For example, the correlation between $\mathrm{BD}$ and width factor (BD-MLD), and the square root of ratios DHI (MLD/BD), and MHI (H/BD) were 0.96, 0.82, and 0.62 respectively.

Table 1 Baseline features of all 31 patients included

\begin{tabular}{lc}
\hline Feature & Value \\
\hline Age, years (mean, SD, range) & $71,6,63-83$ \\
Sex, $n$ (\%) & 9 male $(29 \%)$ \\
Preoperative visual acuity, logMAR & $0.72,0.22,0.30-1$ \\
(mean, SD, range) & \\
Lens status, $n$ (\%) & 26 Phakic $(84 \%)$, \\
& 5 pseudophakic $(16 \%)$ \\
VMA width, $\mu$ m (mean, SD, range) & $323,128,102-596$ \\
MLD (mean, SD, range) & $278,86,80-395$ \\
BD (mean, SD, range) & $580,284,163-1326$ \\
Width factor (mean, SD, range) & $302,243,-130-955$ \\
MHI (mean, SD, range) & $0.72,0.29,0.33-1.69$ \\
DHI (mean, SD, range) & $0.56,0.30,0.21-1.80$ \\
THI (mean, SD, range) & $1.39,0.49,0.49-3$ \\
Macular hole closure at day 28, $n(\%)$ & $11(35 \%)$ \\
VMA release at day 28, $n(\%)$ & $19(61 \%)$ \\
\hline
\end{tabular}

Abbreviations: $\mathrm{BD}$, base diameter; DHI, diameter hole index; MLD, minimum linear diameter; MHI, macular hole index; THI, tractional hole index; VMA, vitreomacular adhesion. 
The characteristics of the patients divided by closure are shown in Table 3.

On univariate analysis, preoperative visual acuity $\geq 0.8$ $(\geq 6 / 36)$ and height were significantly associated with closure; patients with a visual acuity of worse than 6/36 or thicker retinas were less likely to close than those with better vision than $6 / 36$ and thinner retinas. However, from Table 3, it is clear that BD, and its derived variables, was the key factor for predicting closure. Importantly, MLD was not significantly predictive of closure. When all four BD-based variables were fitted into a model using binary logistic regression, width factor (BD-MLD) was the most predictive of outcome. Using multivariate analysis, width factor was significantly associated with closure $(P<0.001)$. The deviance $R^{2}$ was $67.2 \%$ for width factor predicting closure (Figure 1). If the width factor was $<60$, there was a $95 \%$ certainty of closure. If the width factor was 175 , there was a $50 \%$ chance of closure and if the width factor was $>290$ then there was less than a $5 \%$ chance of closure (Figure 2). If BD alone is used, the $R^{2}$ value of the model was lower at $49.3 \%$ and hence less predictive of closure. Using this model, the $95 \%, 50 \%$, and $5 \%$ BD measurement values predictive of closure were 179,424 , and $669 \mu \mathrm{m}$, respectively.

Table 2 Correlations between baseline characteristics

\begin{tabular}{lccc}
\hline & $B D$ & $M L D$ & Height \\
\hline MLD & $0.60^{* * *}$ & & \\
Height & $0.73^{* * *}$ & $0.51^{* *}$ & \\
VMA & $0.37^{*}$ & $0.35^{*}$ & $0.45^{* *}$ \\
\hline
\end{tabular}

Abbreviations: $\mathrm{BD}$, base diameter; $\mathrm{MLD}$, minimum linear diameter; VMA, vitreomacular adhesion.

${ }^{*} P<0.05,{ }^{* *} P<0.01,{ }^{* * *} P<0.001$.
For VMA release, we found that only BD and its derived variables were significantly associated with release and the most predictive factor was $\mathrm{MHI}$ $(P<0.001)$. We found no significant association between the width of VMA and the occurrence of VMA release. The mean VMA width measured $299 \mu \mathrm{m}$ (SE 29) in those with release and $360 \mu \mathrm{m}$ (SE 38) in those without release $(P=0.20)$. Similarly age and lens status were not found to be significantly associated with release. Using binary logistic regression, the deviance $R^{2}$ was $35.2 \%$ for $\mathrm{MHI}$ predicting VMA separation. MLD was again not significantly associated with release $(P=0.23)$.

\section{Discussion}

OCP is the first approved non-vitrectomising technique to close macular holes. When successful, the results can be excellent with $\sim 50 \%$ of patients with closure gaining three or more lines of vision, and avoiding some of the side effects of vitrectomy including cataract production. ${ }^{1,2}$ It is however a relatively expensive treatment with an emerging adverse event profile including retinal detachment, seen in two patients in this series, and acute visual loss with outer retinal dysfunction. ${ }^{14-18}$ It is therefore important to identify the features that could predict success.

The overall closure rate in this series of $35 \%$ is fairly similar to that found in other studies. Importantly, however, we found a morphological feature that was highly predictive of closure, namely, what we termed width factor: the BD minus the MLD. The smaller the $\mathrm{BD}$ diameter was, relative to the MLD, the greater the chance of closure. BD alone was less predictive of closure and importantly MLD on its own was not significantly predictive of closure, although no holes $>400 \mu \mathrm{m}$ in MLD

Table 3 Characteristics of patients by closure

\begin{tabular}{|c|c|c|c|}
\hline & $\begin{array}{l}\text { Closure with OCP } \\
\quad(\mathrm{n}=11)\end{array}$ & $\begin{array}{l}\text { Non closure with OCP } \\
(\mathrm{n}=20)\end{array}$ & P-value \\
\hline Age, years mean (SE, range) & $70.1(1.1,66-78)$ & $71.2(1.7,47-83)$ & 0.6 \\
\hline Sex, $n(\%)$ & 2 male $(18 \%)$ & 7 male $(35 \%)$ & 0.32 \\
\hline Number of patients with preoperative visual acuity $\geq 0.8(20 / 120) \log M A R, n(\%)$ & $1(9 \%)$ & $10(50 \%)$ & 0.02 \\
\hline Lens status, $n(\%)$ & All phakic $(100 \%)$ & 15 phakic $(75 \%)$ & 0.07 \\
\hline VMA width, $\mu$ m mean (SE, range) & $307(32,175-516)$ & $331(32,102-596)$ & 0.64 \\
\hline MLD mean (SE, range) & $258(26,154-395)$ & $289(20,80-395)$ & 0.36 \\
\hline $\mathrm{BD}$ mean $(\mathrm{SE}$, range) & $336(30,163-501)$ & $714(61,265-1326)$ & $<0.001$ \\
\hline Height mean (SE, range) & $309(17,231-396)$ & $381(19,240-538)$ & 0.02 \\
\hline SquRt MHI mean (SE) & $0.98(0.04)$ & $0.76(0.03)$ & $<0.001$ \\
\hline SquRt DHI mean (SE) & $0.89(0.06)$ & $0.64(0.02)$ & $<0.001$ \\
\hline SquRt THI mean (SE) & $1.12(0.04)$ & $1.19(0.05)$ & 0.33 \\
\hline Width factor (BD-MLD) mean (SE, range) & $78(29,-130-182)$ & $425(48,91-955)$ & $<0.001$ \\
\hline
\end{tabular}

Abbreviations: BD, base diameter; DHI, diameter hole index; MLD, minimum linear diameter; MHI, macular hole index; SquRt, square root; THI, tractional hole index; VMA, vitreomacular adhesion. 
were included based on trial results, licensing, and local funding. Similarly, patients with any ERM were excluded as this has been shown to be related to unsuccessful VMA release. $^{8}$

In the MIVI-TRUST trials, 19 out of the 43 with nonsurgical MH closure did not have VMA release by day 28 or month $6 .^{2}$ We did not have any patients who closed without VMA release. We observed eight patients who experienced VMA release without hole closure.

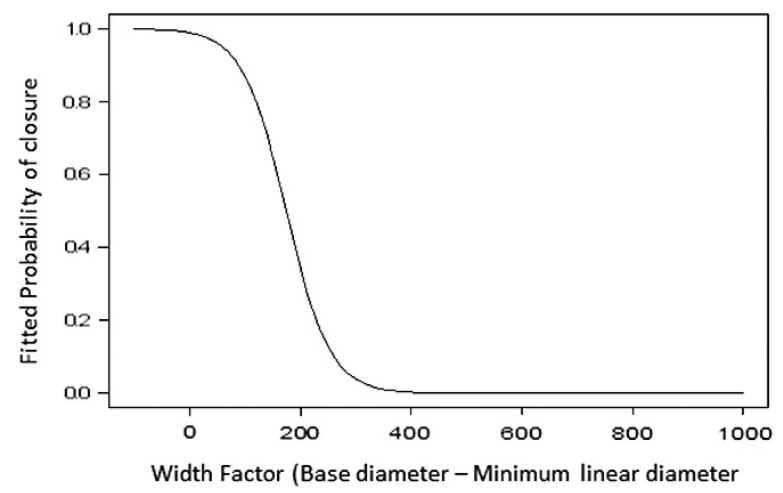

Figure 1 Graph of probability of closure versus width factor.
Presumably, in these patients, other factors have a role in persistent hole opening including tangential traction related to residual vitreous tissue on the ILM, although we have recently shown that this is less after OCP treatment for IMH. ${ }^{19}$ The ILM itself contributes very significantly to the rigidity of the retina ${ }^{20}$ and its removal is known to improve macular hole closure. ${ }^{21}$ Atomic force measurements have shown that ILM stiffness is greater on the retinal side than the vitreous side, accounting for its tendency to scroll upwards as peeled and as such its presence will act to maintain hole opening. ${ }^{22}$ Changes in retinal morphology are known to occur with advancing macular hole formation, such as those recently described by Woon et al and ascribed to the central fovea having a bistable structure. ${ }^{23}$ They observed that BD increases to a far greater extent than MLD as holes enlarge and this may underpin our observation that holes with narrow $\mathrm{BD}$ relative to MLD were most likely to close being at an earlier stage in their development. It may be that an interplay between macular hole size and ILM characteristics in individual patients define whether closure can occur with VMA release alone or indeed the need for ILM peeling with surgery.

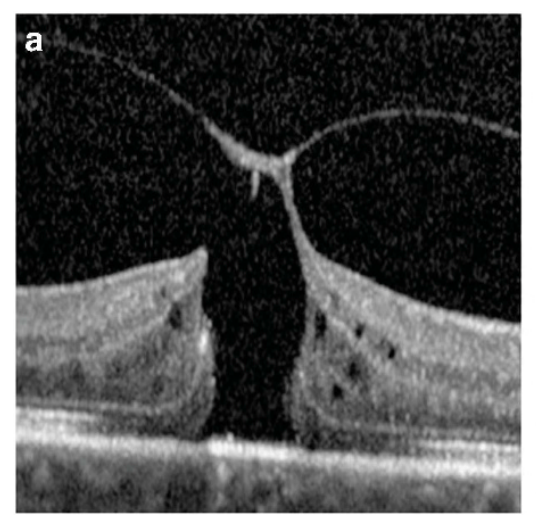

MLD 396, BD 510: Width factor 115

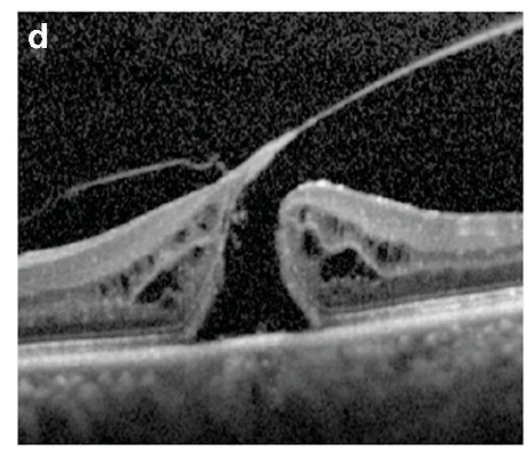

MLD 392, BD 814: Width factor 419

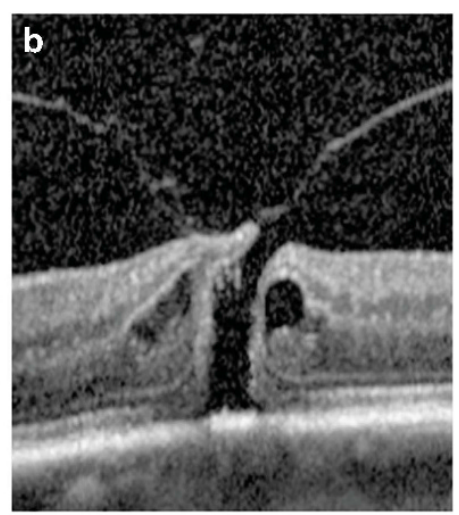

MLD 199, BD 280: Width factor 81

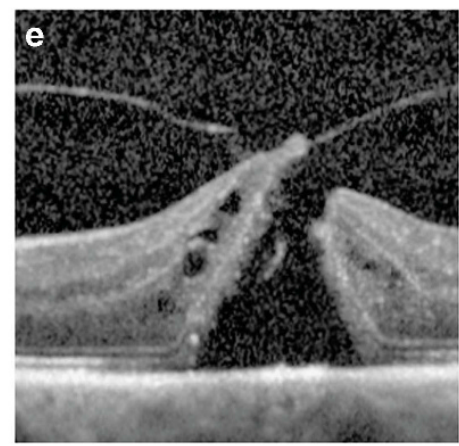

MLD 356, BD 870: Width factor 514

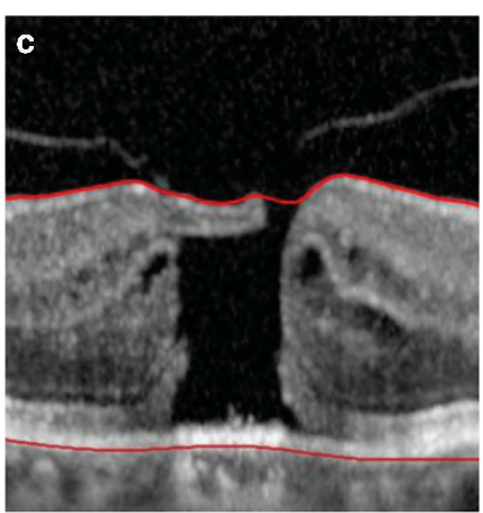

MLD 375, BD 485: Width factor 110

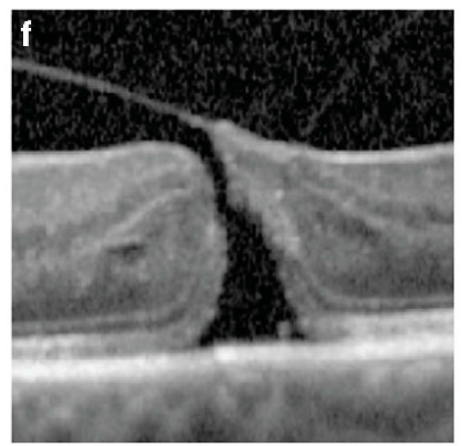

MLD 200, BD 450: Width factor 250

Figure 2 Representative example of three cases with successful primary closure after ocriplasmin (a-c) and three cases without closure $(\mathrm{d}-\mathrm{f})$ with corresponding measurements. 
Another factor derived from BD, MHI was the most predictive variable for VMA release. The narrower the $\mathrm{BD}$ was, relative to the height of the hole, the greater the likelihood of VMA release. MHI has been proposed to represent the putative tangential and anteroposterior vitreomacular traction thought to be acting on the fovea and causing the macular hole to develop. ${ }^{12}$ The association with MHI suggests that holes with greater presumed tractional forces release VMA more readily. By distinction, VMA width that ranged in the study from 102 to $596 \mu \mathrm{m}$ was not predictive of release. Indeed, the case with a $102 \mu \mathrm{m}$ VMA did not release. Moreover, a range of factors will affect local VMA release including the dose of OCP reaching the area of the VMA and local cellular proliferation around the area of VMA not detectable on SD-OCT.

The study has several limitations. Although the sample size in this paper is larger than other independent series, it is still relatively small. However, despite that highly significant effects were found. It is possible that in a much larger study, additional variables would become significant in predicting VMA release and closure. Two different OCT machines were used but both were SD and measurements were made manually by one observer avoiding segmentation differences. ${ }^{24}$ Measurement variability is a known issue however when assessing macular holes and it is possible that the results would not be the same using different machines and observers. ${ }^{25}$ All patients were white Caucasian and the results may not be applicable to other populations. We used macular hole closure as our main end point rather than visual acuity as although the ultimate aim of treatment is to improve vision, closure is a well-defined criteria for success.

In conclusion, we found that the width factor, calculated by subtracting MLD from BD, was highly prognostic for predicting closure in patients with fullthickness IMHs treated with OCP. Further studies to validate this finding are needed.

\section{Summary}

What was known before

- OCP results in vitreomacular attachment release and macular hole closure in a proportion of patients with macular holes $<400 \mu \mathrm{m}$ in MLD. There have been no factors discovered which are highly predictive for successful closure.

\section{What this study adds}

- Width factor, defined as the BD minus the MLD, is highly predictive of closure in patients with macular holes treated with OCP. Patients with macular holes with a width factor of $<60 \mu \mathrm{m}$ have a higher certainty of closure and conversely if the width factor is $>290 \mu \mathrm{m}$, then there is a lower chance of closure.

\section{Conflict of interest}

DHWS, RJH, and IAE declare that they have attended advisory boards for Alcon in connection with ocriplasmin and received travel expenses. $\mathrm{MSH}$ declares he has received travel expenses from Alcon to attend meetings.

\section{References}

1 Stalmans P, Benz MS, Gandorfer A, Kampik A, Girach A, Pakola $S$ et al. Enzymatic vitreolysis with ocriplasmin for vitreomacular traction and macular holes. N Engl J Med 2012; 367: 606-615.

2 Dugel PU, Regillo C, Eliott D. Characterization of anatomic and visual function outcomes in patients with full-thickness macular hole in Ocriplasmin phase 3 trials. Am J Ophthalmol 2015; 160(1): 94-9.e1.

3 Sharma P, Juhn A, Houston SK, Fineman M, Chiang A, Ho A et al. Efficacy of intravitreal ocriplasmin on vitreomacular traction and full-thickness macular holes. Am J Ophthalmol 2015; 159: 861-867.

4 Warrow DJ, Lai MM, Patel A, Raevis J, Berinstein DM. Treatment outcomes and spectral-domain optical coherence tomography findings of eyes with symptomatic vitreomacular adhesion treated with intravitreal ocriplasmin. Am J Ophthalmol 2015; 159: 20-30.

5 Miller JB, Kim LA, Wu DM, Vavvas DG, Eliott D, Husain D. Ocriplasmin for treatment of stage 2 macular holes: early clinical results. Ophthalmic Surg Lasers Imaging Retina 2014; 45: 293-297.

6 Singh RP, Li A, Bedi R, Srivastava S, Sears JE, Ehlers JP et al. Anatomical and visual outcomes following ocriplasmin treatment for symptomatic vitreomacular traction syndrome. Br J Ophthalmol 2014; 98: 356-360.

7 Kim BT, Schwartz SG, Smiddy WE, Doshi RR, Kovach JL, Berrocal AM et al. Initial outcomes following intravitreal ocriplasmin for treatment of symptomatic vitreomacular adhesion. Ophthalmic Surg Lasers Imaging Retina 2013; 44: 334-343.

8 Haller JA, Stalmans P, Benz MS, Gandorfer A, Pakola SJ, Girach A et al. Efficacy of intravitreal ocriplasmin for treatment of vitreomacular adhesion: subgroup analyses from two randomized trials. Ophthalmology 2015; 122: 117-122.

9 Steel DH, Lotery AJ. Idiopathic vitreomacular traction and macular hole: a comprehensive review of pathophysiology, diagnosis, and treatment. Eye 2013; 27(Suppl 1): S1-21.

10 Madi HA, Dinah C, Rees J, Steel DH. The case mix of patients presenting with full-thickness macular holes and progression before surgery: implications for optimum management. Ophthalmologica 2015; 233: 216-221.

11 DeCroos FC, Toth CA, Folgar FA, Pakola S, Stinnett SS, Heydary CS et al. Characterization of vitreoretinal interface disorders using OCT in the interventional phase 3 trials of ocriplasmin. Invest Ophthalmol Vis Sci 2012; 53: 6504-6511.

12 Kusuhara S, Teraoka Escaño MF, Fujii S, Nakanishi Y, Tamura Y, Nagai A et al. Prediction of postoperative visual outcome based on hole configuration by optical coherence tomography in eyes with idiopathic macular holes. Am J Ophthalmol 2004; 138: 709-716.

13 Ruiz-Moreno JM, Staicu C, Piñero DP, Montero J, Lugo F, Amat P. Optical coherence tomography predictive factors for macular hole surgery outcome. Br J Ophthalmol 2008; 92(5): 640-644. 
14 Fahim AT, Khan NW, Johnson MW. Acute panretinal structural and functional abnormalities after intravitreous ocriplasmin injection. JAMA Ophthalmol 2014; 132: 484-486.

15 Johnson MW, Fahim AT, Rao RC. Acute ocriplasmin retinopathy. Retina 2015; 35: 1055-1058.

16 Reiss B, Smithen L, Mansour S. Transient vision loss after ocriplasmin injection. Retina 2015; 35: 1107-1110.

17 Quezada Ruiz C, Pieramici DJ, Nasir M, Rabena M, Avery RL. Severe acute vision loss, dyschromatopsia, and changes in the ellipsoid zone on SD-OCT associated with intravitreal ocriplasmin injection. Retin Cases Brief Rep 2015; 9: 145-148.

18 Chang JS, Smiddy WE. Cost evaluation of surgical and pharmaceutical options in treatment for vitreomacular adhesions and macular holes. Ophthalmology 2014; 121(9): 1720-1726.

19 Steel DH, Sandinha MT, White K. The plane of vitreoretinal separation and results of vitrectomy surgery in patients given ocriplasmin for idiopathic macular hole. Invest Ophthalmol Vis Sci 2015; 56(6): 4038-4044.

20 Wollensak G, Spoerl E, Grosse G, Wirbelauer C. Biomechanical significance of the human internal limiting lamina. Retina 2006; 26(8): 965-968.
21 Spiteri Cornish K, Lois N, Scott NW, Burr J, Cook J, Boachie C et al. Vitrectomy with internal limiting membrane peeling versus no peeling for idiopathic full-thickness macular hole. Ophthalmology 2014; 121(3): 649-655.

22 Henrich PB, Monnier CA, Halfter W, Haritoglou C, Strauss RW, Lim RY et al. Nanoscale topographic and biomechanical studies of the human internal limiting membrane. Invest Ophthalmol Vis Sci 2012; 53(6): 2561-2570.

23 Woon WH, Greig D, Savage MD, Wilson MC, Grant CA, Mokete $\mathrm{B}$ et al. Movement of the inner retina complex during the development of primary full-thickness macular holes: implications for hypotheses of pathogenesis. Graefes Arch Clin Exp Ophthalmol 2015; 253: 2103-2109.

24 Heussen FM, Ouyang Y, McDonnell EC, Narala R, Ruiz-Garcia H, Walsh AC et al. Comparison of manually corrected retinal thickness measurements from multiple spectral-domain optical coherence tomography instruments. Br J Ophthalmol 2012; 96: 380-385.

25 Banerjee P, Elgohary M, Wickham L, Charteris D. Measuring macula hole size on optical coherence tomography; inter and intra observer agreement. Invest Ophthalmol Vis Sci 2013; 54(15): 3612. 